Cite this: Phys. Chem. Chem. Phys., 2013, 15, 17263

Received 31st July 2013, Accepted 19th August 2013 DOI: $10.1039 / \mathrm{c} 3 \mathrm{cp} 53243 \mathrm{~g}$

www.rsc.org/pccp

\title{
Elucidating the backbone conformation of photoswitchable foldamers using vibrational circular dichroism
}

\author{
Sérgio R. Domingos, ${ }^{a}$ Steven J. Roeters, ${ }^{a}$ Saeed Amirjalayer, ${ }^{a}$ Zhilin Yu, ${ }^{b}$ \\ Stefan Hecht ${ }^{* b}$ and Sander Woutersen ${ }^{* a}$
}

\begin{abstract}
The backbone conformation of amphiphilic oligo(azobenzene) foldamers is investigated using vibrational circular dichroism (VCD) spectroscopy on a mode involving the stretching of the $\mathrm{N}=\mathrm{N}$ bonds in the backbone. From denaturation experiments, we find that the VCD response in the helical conformation arises mainly from through-space interaction between the $\mathrm{N}=\mathrm{N}$-stretch transition-dipole moments, so that the coupled-oscillator model can be used to predict the VCD spectrum associated with a particular conformation. Using this approach, we elucidate the origin of the VCD signals in the folded conformation, and can assign the observed partial loss of VCD signals upon photo-induced unfolding to specific conformational changes. Our results show that the $\mathrm{N}=\mathrm{N}$-stretch $\mathrm{VCD}$ response provides an excellent probe of the helical conformation of the $\mathrm{N}=\mathrm{N}$ bonds in this type of switchable molecular system.
\end{abstract}

\section{Introduction}

Molecular systems with the ability to undergo conformational changes triggered by light have inspired the design of lightactivated molecular machinery. ${ }^{1-6}$ An interesting class of such artificial molecular architectures is formed by photoswitchable amphiphilic oligo(azobenzene) foldamers, which can be manipulated to undergo a helix-coil conformational transition upon photoisomerization around the $\mathrm{N}=\mathrm{N}$ bonds, with extraordinary folding-unfolding efficiencies. ${ }^{2,7-9}$ The backbone of these foldamers consists of azobenzene monomeric units, which can be assembled to obtain molecular systems of various lengths (Fig. 1). The photo-induced trans-to-cis isomerization triggers a conformational change in the backbone of the $X_{n}$ azo helix (where $X$ denotes the number of phenylene units, and $n$ indicates the number of azobenzene moieties), causing it to unfold. ${ }^{7-9}$ Refolding to the helical conformation can be achieved by irradiation with visible light or alternatively by thermal relaxation. ${ }^{7-9}$

As yet, the folding and unfolding of synthetic foldamers are generally investigated using electronic circular dichroism (CD), ${ }^{11}$ which provides a rather indirect probe of the helical backbone conformation. Here, we investigate the conformation and photoinduced conformational changes in azo-foldamers using vibrational circular dichroism (VCD) spectroscopy ${ }^{12,13}$ on a vibrational

\footnotetext{
${ }^{a}$ Van 't Hoff Institute for Molecular Sciences, University of Amsterdam, Science Park 904, 1098 XH, Amsterdam, The Netherlands. E-mail: s.woutersen@uva.nl

${ }^{b}$ Department of Chemistry, Humboldt-Universität zu Berlin, Brook-Taylor-Strasse 2, 12489 Berlin, Germany. E-mail: sh@chemie.hu-berlin.de
}
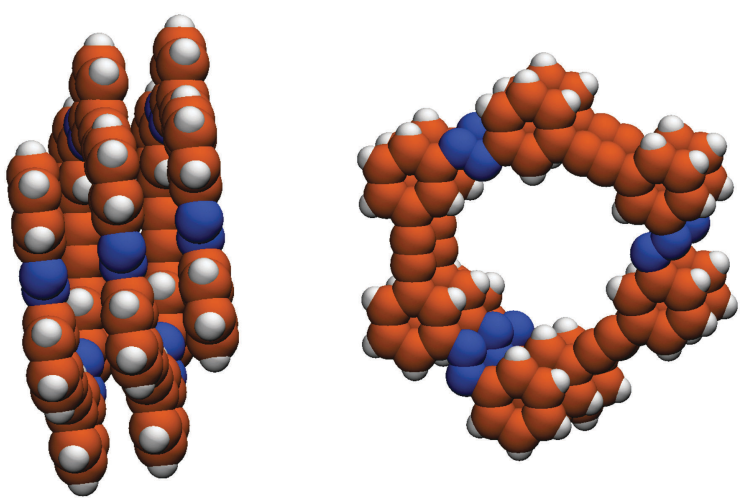

Fig. 1 Side view along the $y$ axis (left) and top view along the $z$ axis (right) of the backbone structure of an $X_{n}$ azobenzene foldamer in its folded conformation. In this case, $X=14$ and $n=7$. The structure is optimized with the $M M 2^{10}$ force field.

mode involving the $\mathrm{N}=\mathrm{N}$-stretch and in-plane vibrations of the aromatic rings of the azobenzene units in the foldamer backbone. In the last decade VCD has emerged as a powerful structure-resolving tool to study chiral molecules. In most cases, the interpretation of VCD spectra relies on complementary density-functional theory (DFT) calculations, and combined with such calculations VCD provides detailed information on the configuration and conformation of chiral molecules in the condensed phase. ${ }^{14-23}$ In particular, studies that combine VCD spectroscopy and $a b$ initio calculations show convincing results regarding the preferred secondary structures of foldamers. ${ }^{24-26}$ As an alternative to spectral interpretation using DFT calculations, 
the simpler, semiquantitative coupled-oscillator method, in which the molecular vibrations are treated as localized, interacting transition dipoles, is attractive because of its transparency and computational ease, ${ }^{27-30}$ in particular for larger systems such as foldamers. Since we find that the $\mathrm{N}=\mathrm{N}$-stretch VCD signal of azo-foldamers arises predominantly from throughspace interaction between the $\mathrm{N}=\mathrm{N}$-stretch transition-dipole moments, we can use this coupled-oscillator approach to interpret the observed foldamer VCD spectra. We find that the $\mathrm{N}=\mathrm{N}$ groups give rise to unique VCD signatures, which not only provide direct access to the twist sense of the helical structure, but also contain more structural information than the conventional (electronic) CD spectrum.

\section{Materials and methods}

The synthesis and purification of the foldamers have been reported elsewhere. ${ }^{7}$ Fourier-transform infrared (FTIR) and VCD spectra were obtained using a Bruker Vertex 70 spectrometer in combination with a PMA 50 module for polarization modulation measurements. Samples of the $\mathbf{1 4}_{\mathbf{7}}$ foldamer were prepared in $\mathrm{CD}_{3} \mathrm{CN}$ and $\mathrm{CD}_{2} \mathrm{Cl}_{2}$ and kept in sealed infrared cells with $\mathrm{CaF}_{2}$ windows. Photo-switching of the $\mathbf{1 4}_{\mathbf{7}}$ foldamer was performed using a $450 \mathrm{~W}$ Xe lamp (Müller Elektronik) and a notch filter with $T=12 \%$ at $\lambda=358 \mathrm{~nm}(\mathrm{FWHM}=45 \mathrm{~nm})$. The cartesian coordinates of the nitrogen atoms in the backbone of the $\mathbf{1 4}_{\mathbf{7}}$ foldamer were retrieved from an optimized structure obtained using the MM2 $2^{10}$ force field (Fig. 1).

\section{Mode assignment}

The folded conformation of a $\mathbf{1 4}_{\mathbf{7}}$ azo-foldamer is structurally organized as follows: an alternating sequence of seven azobenzene units is connected through ethynylene bridges. The side chains consist of oligo(ethylene glycol) moieties, which are attached to the main chain azobenzene phenyl rings and thus provide the necessary solvophobic driving force for folding in a polar medium. ${ }^{7}$ In addition, the presence of the side-chains causes a symmetry breaking around the $\mathrm{N}=\mathrm{N}$ bond. This lower symmetry as compared to that of non-substituted azobenzene gives rise to infrared activity of the vibrational mode involving mainly the stretching of the $\mathrm{N}=\mathrm{N}$ bond. For simplicity, we will from now on refer to this mode (which also involves the phenyl ring vibration) as the $\mathrm{N}=\mathrm{N}$-stretch mode. The infrared activity of the $\mathrm{N}=\mathrm{N}$-stretching mode in such non-symmetrically substituted trans-azobenzene systems has been convincingly demonstrated for a series of compounds in a recent combined experimental and theoretical study. ${ }^{31}$ We confirmed our assignment of the IR band at $\approx 1450 \mathrm{~cm}^{-1}$ to this mode by quantumchemical calculations on an isolated substituted azobenzene unit (Fig. 2). The geometry optimization and calculation of harmonic vibrational frequencies were performed at the DFT/ BLYP/6-31G(d) ${ }^{35-37}$ level of theory for a simplified model of the system, where we substitute the oligo(ethylene glycol) branches by ethyl-ester groups. Fig. 2 displays the calculated displacement vectors and the direction of the transition-dipole moment
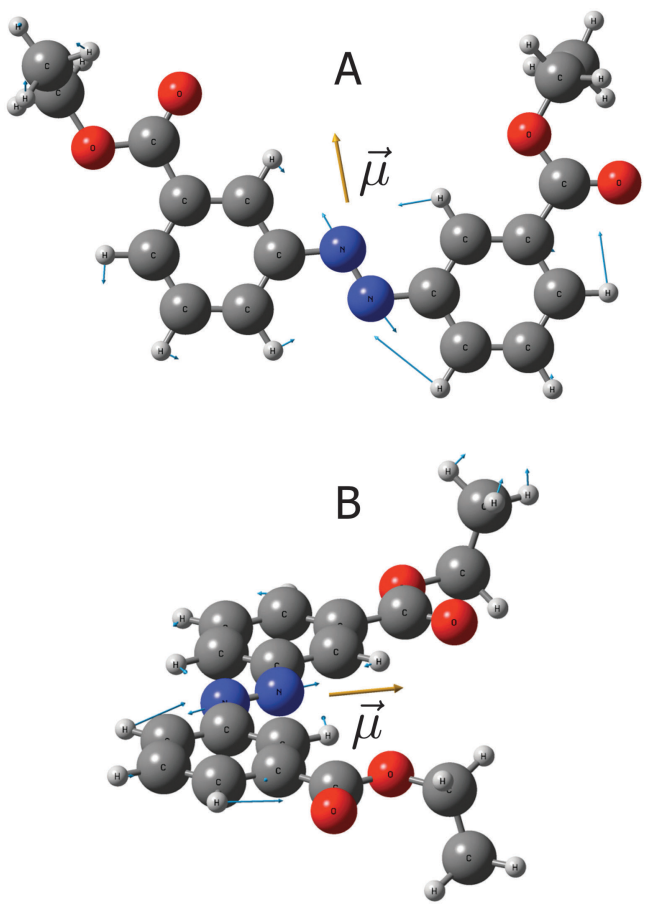

Fig. 2 Displacement vectors (in blue) and transition dipole moment vector $\left(\vec{\mu}_{i}\right.$ in yellow) for the $\mathrm{N}=\mathrm{N}$-stretching mode $\left(\nu=1450 \mathrm{~cm}^{-1}\right)$ of asymmetrically substituted azobenzene calculated at the DFT/BLYP/6-31G(d) level of theory. A - top view; B - side view.

for the $\mathrm{N}=\mathrm{N}$-stretching mode. We find the transition dipole to be approximately collinear $\left(\approx 15^{\circ}\right)$ with the $\mathrm{N}=\mathrm{N}$ bond.

\section{$4 \mathrm{~N}=\mathrm{N}$-stretch VCD spectra}

Initially we investigate to what extent the observed VCD signal arises from through-space interactions between the $\mathrm{N}=\mathrm{N}$-stretch transition-dipole moments. Previous studies with electronic CD have shown that the helical content of $\mathbf{1 4}_{7}$ in $\mathrm{CD}_{3} \mathrm{CN}$ solution progressively vanishes upon addition of $\mathrm{CDCl}_{3}$ to the solution due to the reduction of the solvophobic effect. We therefore measured the IR and VCD spectra of a $10^{-4} \mathrm{M}$ solution of $\mathbf{1 4}_{7}$ in $\mathrm{CD}_{3} \mathrm{CN}$ and $\mathrm{CD}_{2} \mathrm{Cl}_{2}$ (Fig. $3 ; \mathrm{CD}_{2} \mathrm{Cl}_{2}$ rather than $\mathrm{CDCl}_{3}$ was used as a solvent because of strong infrared absorption of $\mathrm{CDCl}_{3}$ in the $\mathrm{N}=\mathrm{N}$-stretch region). We find no $\mathrm{N}=\mathrm{N}$-stretch VCD signal arising from the denatured, optically-inactive conformation of the foldamer in apolar media. This proves that the VCD signals observed for the folded conformation (the black solid line in Fig. 3) arise mainly from the helical arrangement and through-space coupling of the $\mathrm{N}=\mathrm{N}$-stretch vibrational chromophores.

\subsection{Coupled-oscillator model for the $\mathrm{N}=\mathrm{N}$-stretch modes}

To calculate the vibrational frequencies and the vibrational circular dichroism of a helical azo-foldamer containing $n \mathrm{~N}=\mathrm{N}$ groups using the coupled-oscillator model, we use the formalism derived by Diem et $a l^{32}$ Due to the interaction between the $\mathrm{N}=\mathrm{N}$-stretch modes, the $\mathrm{N}=\mathrm{N}$-stretch normal modes will be 


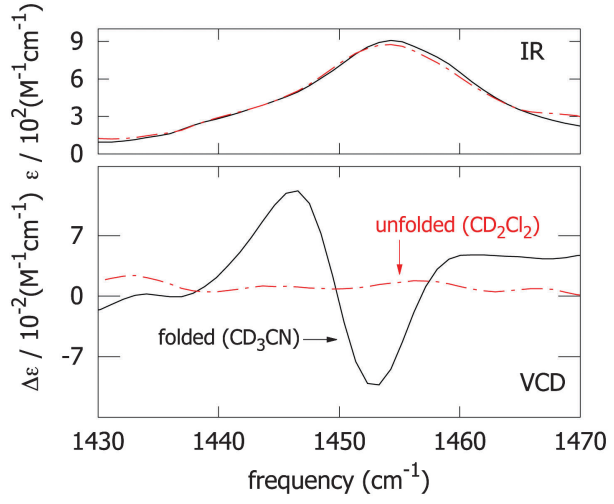

Fig. 3 Infrared absorption (upper panel) and VCD (lower panel) spectra of the 147 azo-foldamer in the folded $\left(\mathrm{CD}_{3} \mathrm{CN}\right.$, solid black lines) and unfolded conformation $\left(\mathrm{CD}_{2} \mathrm{Cl}_{2}\right.$, dashed red lines).

delocalized over the helix, i.e., they will involve the vibrational motion of more than one chromophore. These delocalized normal modes are the eigenstates of the excitonic Hamiltonian

$$
H=\left[\begin{array}{ccccc}
\nu_{0} & J_{12} & J_{13} & \ldots & J_{1 n} \\
J_{21} & \nu_{0} & J_{23} & \ldots & J_{2 n} \\
J_{31} & J_{32} & \nu_{0} & \ldots & J_{3 n} \\
\vdots & \vdots & \vdots & \ddots & \vdots \\
J_{n 1} & J_{n 2} & J_{n 3} & \ldots & \nu_{0}
\end{array}\right]
$$

where $\nu_{0}$ is the frequency of an unperturbed $\mathrm{N}=\mathrm{N}$-stretch mode, $J_{i j}$ the coupling between $\mathrm{N}=\mathrm{N}$-stretch modes $i$ and $j$. The Hamiltonian $H$ is a symmetric (Hermitian and real) matrix so that $J_{i j}=J_{j i}$. The interaction between the $\mathrm{N}=\mathrm{N}$ groups is described by transition-dipole coupling:

$$
J_{i j}=\frac{1}{4 \pi \varepsilon_{0}}\left[\frac{\vec{\mu}_{i} \cdot \vec{\mu}_{j}}{\left|\vec{r}_{i j}\right|^{3}}-\frac{3\left(\vec{\mu}_{i} \cdot \vec{r}_{i j}\right)\left(\vec{\mu}_{j} \cdot \vec{r}_{i j}\right)}{\left|\vec{r}_{i j}\right|^{5}}\right],
$$

where $\vec{r}_{i j}$ is the distance vector between dipoles $\vec{\mu}_{i}$ and $\vec{\mu}_{j}$ (Fig. 4). Note that the meta-linkages between the individual azobenzene repeated units prevent any through-bond coupling due to crossconjugation.

The dipole-dipole interaction lifts the degeneracy of the $\mathrm{N}=\mathrm{N}$-stretching frequencies, and gives rise to as many delocalized normal modes as there are interacting $\mathrm{N}=\mathrm{N}$ oscillators. The vibrational frequencies of these delocalized normal modes (excitons) are the eigenvalues of the excitonic Hamiltonian matrix $H$. The dipole $(D)$ and rotational $(R)$ strengths of the $k$ th exciton are given by $^{32}$

$$
D_{k}=\sum_{i=1}^{N} \sum_{j=1}^{N} b_{i k} b_{j k}\left(\vec{\mu}_{i} \cdot \vec{\mu}_{j}\right)
$$

and

$$
R_{k}=-\left(\pi \nu_{0} / c\right) \sum_{i=1}^{N} \sum_{j>1}^{N} b_{i k} b_{j k}\left[\vec{r}_{i j} \cdot \vec{\mu}_{i} \times \vec{\mu}_{j}\right]
$$

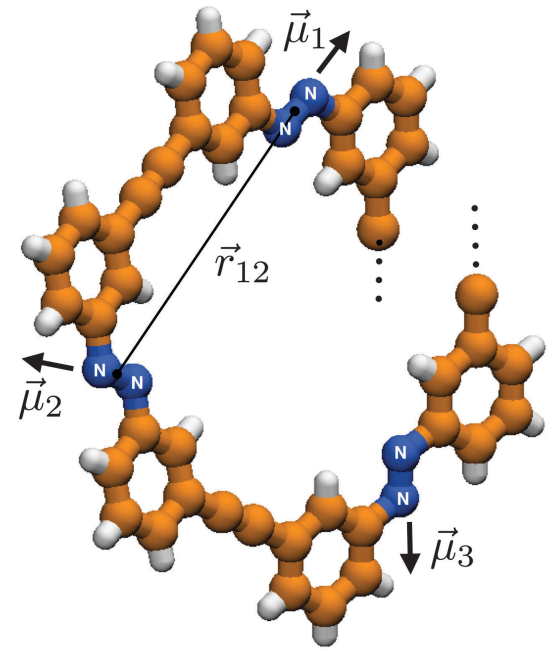

Fig. 4 Schematic representation of an $X_{n}$ foldamer backbone with three monomeric $\mathrm{N}=\mathrm{N}$ units. Each transition dipole is considered to be collinear with the respective $\mathrm{N}=\mathrm{N}$ bond.

where $c$ is the velocity of light in vacuum, and $b_{i j}$ are the components of the eigenvectors of the Hamiltonian. The infrared absorption and vibrational circular dichroism spectra are calculated using eqn (3) and (4), respectively, and convoluted with a Lorentzian band profile.

\section{$4.2 \quad 147$ azo-foldamer}

In Fig. 5a and b (solid lines) we show experimental FTIR and VCD spectra of the $\mathbf{1 4}_{7}$ azo-foldamer, respectively, in the $\mathrm{N}=\mathrm{N}$-stretch

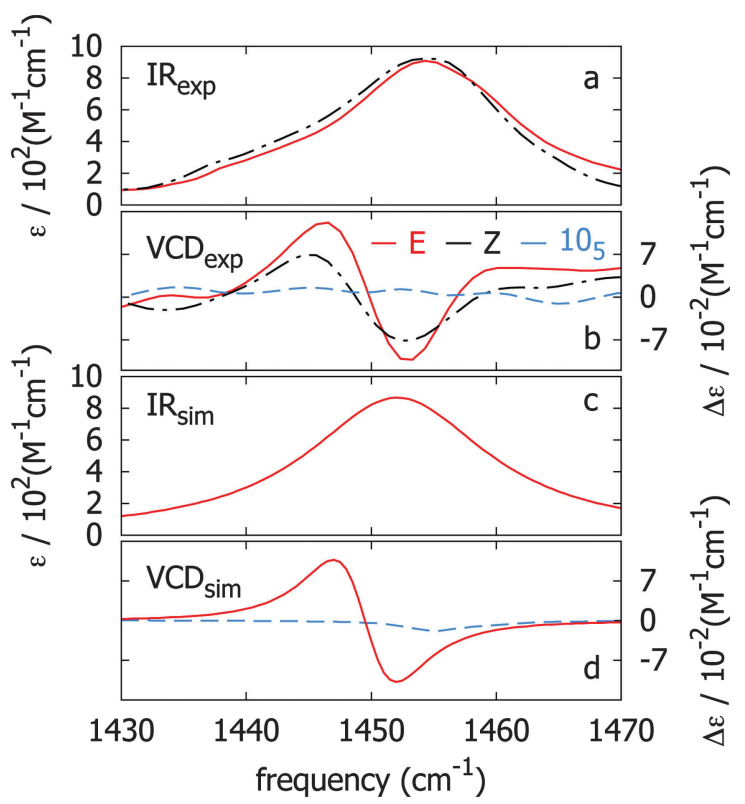

Fig. 5 Observed $(a, b)$ and calculated $(c, d)$ infrared absorption and VCD spectra of the $\mathrm{N}=\mathrm{N}$-stretching mode for the $\mathbf{1 4}_{\mathbf{7}}$ azo-foldamer in its folded conformation ( $E$ form, red curves). Infrared absorption and VCD spectra of the $1_{7}$ azofoldamer upon photo-excitation are depicted in dashed lines ( $Z$ form, black curves). Observed (b) and calculated (d) VCD spectra of a native $10_{5}$ azo-foldamer (dashed blue curves). 
spectral region. Using the experimental infrared absorption spectrum (Fig. 5a, solid line), we determine the magnitude of $\vec{\mu}$ from the integrated absorption coefficient $A=\int \varepsilon(\nu) \mathrm{d} \nu$. This value is used to calculate the coupling terms in eqn (2) and to construct the Hamiltonian with solely $\nu_{0}$ as a free parameter. Fig. $5 \mathrm{c}$ and $\mathrm{d}$ show simulated infrared absorption and VCD spectra (with $|\vec{\mu}|=0.12$ Debye), respectively, for the $\mathrm{N}=\mathrm{N}$-stretch modes calculated using the coupled-oscillator formalism described above. The experimental VCD pattern, which exhibits a characteristic bisignate signal (positive couplet), is nicely reproduced in the simulated spectrum. The three most IR-intense calculated eigenmodes have vibrational frequencies of 1452,1451 and $1450 \mathrm{~cm}^{-1}$. The remaining four predicted eigenmodes have extremely weak intensities (less than $5 \%$ of the total intensity) and are therefore neglected in the following analysis.

Fig. 6 displays the three most intense normal modes as color coding on schematic representations of the $\mathbf{1 4}_{\mathbf{7}}$ backbone structure. The color code is used to illustrate the relative amplitudes (eigenvector coefficients $b_{i j}$ ) of the $\mathrm{N}=\mathrm{N}$ oscillators in the eigenmodes that constitute the VCD spectrum (Fig. 5d). Eigenmode I (Fig. 6-I), which gives rise to the negative VCD peak $\left(\approx 1452 \mathrm{~cm}^{-1}\right)$, has its origin in a strong coupling (with negative amplitudes) of the three vertically aligned $\mathrm{N}=\mathrm{N}$ units (terminal and central units), while the interior units are not strongly involved. On the other hand,

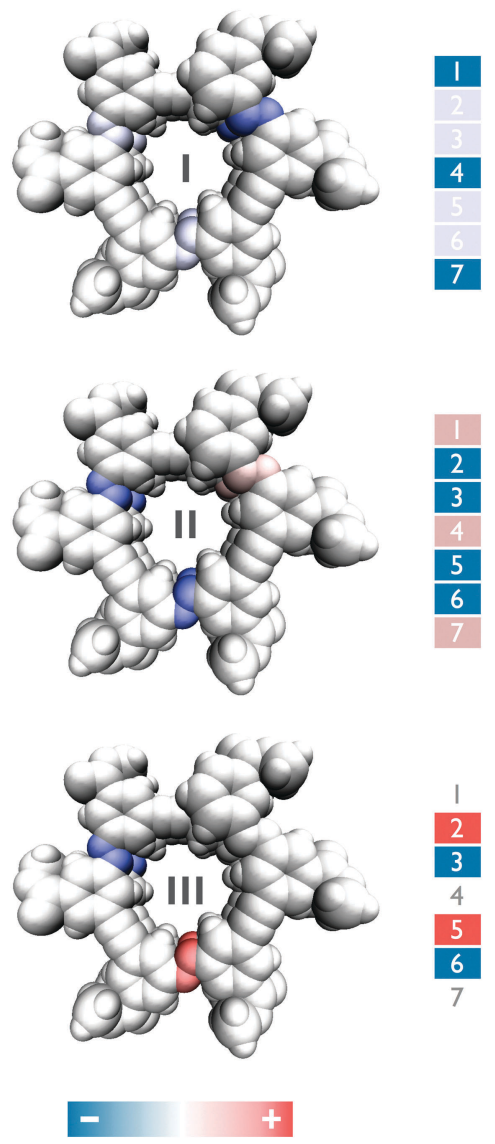

Fig. 6 Schematic representation of the relative amplitudes (eigenvector coefficients $\left.b_{i j}\right)$ of the $\mathrm{N}=\mathrm{N}$ oscillators in the eigenmodes I- $\left(1452 \mathrm{~cm}^{-1}\right), \| I-\left(1451 \mathrm{~cm}^{-1}\right)$ and III $-\left(1450 \mathrm{~cm}^{-1}\right)$ respectively. eigenmode II (Fig. 6-II), which gives rise to the positive VCD peak $\left(\approx 1448 \mathrm{~cm}^{-1}\right.$ ), originates from strong coupling (with positive amplitudes) of the terminal and central $\mathrm{N}=\mathrm{N}$ units (as opposite to eigenmode I) in addition to a strong coupling (with negative amplitudes) between the remaining interior $\mathrm{N}=\mathrm{N}$ groups. Finally, eigenmode III arises from strong contributions with opposite signs from the interior $\mathrm{N}=\mathrm{N}$ units (the $\mathrm{N}=\mathrm{N}$ units involved are depicted with the same color code in Fig. 6), while both terminal and central units have negligible contributions (Fig. 6-III).

It is thus clear that the most intense VCD peaks arise from normal modes involving triply stacked terminal $\mathrm{N}=\mathrm{N}$ groups. This is confirmed by measurement on a $\mathbf{1 0}_{5}$ foldamer which lacks such triple-stacked arrangement. The VCD spectrum of a native-helical $\mathbf{1 0}_{\mathbf{5}}$ foldamer is shown in Fig. $5 \mathrm{~b}$ (dashed blue curve). The lack of signal intensity is consistent with a helical conformation lacking triple-stacked $\mathrm{N}=\mathrm{N}$ units when compared with that of the $\mathbf{1 4}_{\mathbf{7}}$ foldamer (with $7 \mathrm{~N}=\mathrm{N}$ units). Moreover, in Fig. 5d (dashed blue curve) we show the simulated VCD spectrum of a $\mathbf{1 0}_{\mathbf{5}}$ foldamer obtained with $|\vec{\mu}|=0.12$ Debye as an input parameter in the model. The signal magnitudes are extremely weak compared with those of the $\mathbf{1 4}_{7}$ foldamer, which confirms our conclusion that the VCD arises mainly from the triple-stacked $\mathrm{N}=\mathrm{N}$ bonds.

Clearly, the coupled-oscillator formalism applied to the backbone $\mathrm{N}=\mathrm{N}$ units successfully describes the observed features in the VCD spectrum of the $\mathbf{1 4}_{\mathbf{7}}$ azo-foldamer. The good agreement of simulated and observed spectral features confirms the conclusions from the previously reported ECD spectra regarding the native-folded conformation of the azo-foldamer backbone. ${ }^{7-9}$ The good agreement between the observed and the modeled VCD spectra furthermore nicely corroborates the right-handed helix twist sense (P-helicity), which was previously assigned based on the shape of the exciton couplet. ${ }^{33,34}$

\subsection{Photo-induced $14_{7}$ unfolding}

To determine the effect of azobenzene $E \rightarrow Z$ photoisomerization on the VCD response we irradiated a $10^{-4} \mathrm{M}$ solution of $\mathbf{1 4}_{7}$ in $\mathrm{CD}_{3} \mathrm{CN}$ at $\lambda=358 \mathrm{~nm}$ using a $500 \mathrm{~W}$ Xe lamp equipped with a notch filter for a period of $24 \mathrm{~h}$ to assure reaching the photostationary state (PSS). Upon irradiation, the $\mathbf{1 4}_{7}$ azo-foldamer undergoes a helix-coil transition, leading to a PSS in which a fraction of the $\mathrm{N}=\mathrm{N}$ units is in the $Z$-configuration, leading to partial denaturation of the folded helical state. Previous studies have shown that in the PSS approximately $40 \%$ of the $\mathrm{N}=\mathrm{N}$ units that are in the $Z$-form belong to the termini. ${ }^{7}$ The IR and VCD spectra of the PSS are shown in Fig. $5 \mathrm{a}$ and $\mathrm{b}$ (dashed lines). While the IR spectrum shows only a slight red-shift, the VCD couplet is reduced in intensity by approximately $50 \%$. This decrease in signal magnitude corroborates the previous conclusions based on electronic circular dichroism experiments, ${ }^{7}$ where the decrease in signal intensity was assigned to the loss of excess helicity of the azo-foldamer. The observed decrease in intensity of the VCD bisignate signal (Fig. 5b) is consistent with the predicted role of eigenmodes I and II as local probes in the coupling of the $\mathrm{N}=\mathrm{N}$ termini and core units, which is partially disrupted upon photoisomerization. Moreover, the partial denaturation of the foldamer, i.e., the reorientation of the termini and subsequent loss of helical content, effectively leads to the 
formation of a $\mathbf{1 0}_{5}$-type foldamer, in which the backbone still adopts a helical structure, but now with fewer transoid backbone $\mathrm{N}=\mathrm{N}$ units. This observation is in agreement with the unfolding mechanism proposed in which unfolding occurs predominantly starting from the termini of the foldamer. ${ }^{7}$

\section{Conclusion}

Our results demonstrate that VCD can be used to probe conformational changes during the folding and unfolding of azobenzene foldamers. The fact that the measurements can be interpreted using a simple coupled-oscillator approach ensures a straightforward relationship between observed VCD spectra and the foldamer conformation. Much more detailed information can be obtained from VCD measurements on foldamers in which specific $\mathrm{N}=\mathrm{N}$ bonds have been isotope-labeled. These labeled $\mathrm{N}=\mathrm{N}$ bonds will have a different local-mode stretching frequency, and will be spectrally isolated from the other $\mathrm{N}=\mathrm{N}$ bonds. They can therefore be described by a smaller (ideally $2 \times 2$, if two $\mathrm{N}=\mathrm{N}$ bonds are labeled) Hamiltonian. Such experiments should make it possible to probe conformational changes at specific sites in the foldamer backbone, and the interpretation should be just as straightforward as for the unlabeled foldamers studied here.

\section{Acknowledgements}

The authors gratefully acknowledge Michiel F. Hilbers, Hans J. Sanders, Jan-Hein Hooijschuur and Freek Ariese for technical support. We would also like to thank Wybren Jan Buma for critically reading the manuscript. S.R.D. acknowledges financial support from the Portuguese Foundation for Science and Technology (FCT) under the fellowship SFRH/BD/48295/2008. S.A. would like to thank the Deutsche Akademie der Naturforscher Leopoldina German National Academy of Sciences for a Leopoldina research fellowship (grant number LPDS 2011-18). S.H. gratefully acknowledges generous support from the German Research Foundation (DFG via SFB 658), the European Research Council (ERC) via ERC2012-STG 308117 (Light4Function), and the European Science Foundation (ESF via P2M). S.W. acknowledges the European Research Council (ERC) for funding through Grant No. 210999.

\section{References}

1 G. Guichard and I. Huc, Chem. Commun., 2011, 47, 5933-5941.

2 D. Bléger, Z. Yu and S. Hecht, Chem. Commun., 2011, 47, 12260-12266.

3 A. Gopal, M. Hifsudheen, S. Furumi, M. Takeuchi and A. Ajayaghosh, Angew. Chem., Int. Ed., 2012, 51, 10505-10509.

4 X. Yan, F. Wang, B. Zheng and F. Huang, Chem. Soc. Rev., 2012, 41, 6042-6065.

5 S. Braun, M. Bockmann and D. Marx, ChemPhysChem, 2012, 13, 1440-1443.

6 M. R. Panman, P. Bodis, D. J. Shaw, B. H. Bakker, A. C. Newton, E. R. Kay, A. M. Brouwer, W. J. Buma, D. A. Leigh and S. Woutersen, Science, 2010, 328, 1255-1258.

7 Z. Yu and S. Hecht, Angew. Chem., Int. Ed., 2011, 50, 1640-1643.
8 Z. Yu and S. Hecht, Chem.-Eur. J., 2012, 18, 10519-10524.

9 Z. Yu, S. Weidner, T. Risse and S. Hecht, Chem. Sci., 2013, DOI: 10.1039/C3SC51664D.

10 N. L. Allinger, J. Am. Chem. Soc., 1977, 99, 8127-8134.

11 R. B. Prince, L. Brunsveld, E. W. Meijer and J. S. Moore, Angew. Chem., Int. Ed., 2000, 39, 228-230.

12 L. A. Nafie, T. A. Keiderling and P. J. Stephens, J. Am. Chem. Soc., 1976, 98, 2715-2723.

13 P. J. Stephens, J. Phys. Chem., 1985, 89, 748-752.

14 T. B. Freedman, X. Cao, R. K. Dukor and L. A. Nafie, Chirality, 2003, 15, 743-758.

15 S. R. Domingos, M. R. Panman, B. H. Bakker, F. Hartl, W. J. Buma and S. Woutersen, Chem. Commun., 2012, 48, 353-355.

16 S. R. Domingos, P. S. P. Silva, W. J. Buma, M. H. Garcia, N. C. Lopes, J. A. Paixão, M. R. Silva and S. Woutersen, J. Chem. Phys., 2012, 136, 134501.

17 P. J. Stephens, F. J. Devlin and J.-J. Pan, Chirality, 2008, 20, 643-663.

18 C. Merten and Y. Xu, ChemPhysChem, 2013, 14, 213-219.

19 C. Merten and Y. Xu, Angew. Chem., Int. Ed., 2013, 52, 2073-2076.

20 T. J. Measey and R. Schweitzer-Stenner, J. Am. Chem. Soc., 2011, 133, 1066-1076.

21 E. De Gussem, P. Bultinck, M. Feledziak, J. MarchandBrynaert, C. V. Stevens and W. Herrebout, Phys. Chem. Chem. Phys., 2012, 14, 8562-8571.

22 D. Kurouski, R. K. Dukor, X. F. Lu, L. A. Nafie and I. K. Lednev, Biophys. J., 2012, 103, 522-531.

23 V. P. Nicu, E. Debie, W. Herrebout, B. van der Veken, P. Bultinck and E. J. Baerends, Chirality, 2010, 21, E287-E297.

24 T. A. Martinek, I. M. Mandity, L. Fulop, G. K. Toth, E. Vass, M. Hollosi, E. Forro and F. Fulop, J. Am. Chem. Soc., 2006, 128, 13539-13544.

25 L. Ducasse, F. Castet, A. Fritsch, I. Huc and T. Buffeteau, J. Phys. Chem. A, 2007, 111, 5092-5098.

26 G. Longhi, S. Abbate, F. Lebon, N. Castellucci, P. Sabatino and C. Tomasini, J. Org. Chem., 2012, 77, 6033-6042.

27 R. Schweitzer-Stenner, J. Phys. Chem. B, 2004, 108, 16965.

28 R. Schweitzer-Stenner, F. Eker, K. Griebenow, X. Cao and L. A. Nafie, J. Am. Chem. Soc., 2004, 126, 2768-2776.

29 T. Measey and R. Schweitzer-Stenner, Chem. Phys. Lett., 2005, 408, 123-127.

30 E. Schwartz, S. R. Domingos, A. Vdovin, M. Koepf, W. J. Buma, J. J. L. M. Cornelissen, A. E. Rowan, R. J. M. Nolte and S. Woutersen, Macromolecules, 2010, 43, 7931-7935.

31 N. Biswas and S. Umapathy, J. Phys. Chem. A, 2000, 104, 2734-2745.

32 T. Xiang, D. J. Goss and M. Diem, Biophys. J., 1993, 65, 1255-1261. 33 N. Berova, K. Nakanishi and R. W. Woody, Circular Dichroism: Principles and Applications, Wiley, New York, 2nd edn, 2000.

34 D. A. Lightner and J. E. Gurst, Organic Conformational Analysis and Stereochemistry from Circular Dichroism Spectroscopy, Wiley, New York, 2000, p 423, and references therein.

35 M. J. Frisch et al., Gaussian 09, Revision C.02, Inc., Wallingford, CT, 2009.

36 A. D. Becke, Phys. Rev. A, 1988, 38, 3098-3100.

37 C. Lee, W. Yang and R. G. Parr, Phys. Rev. B, 1988, 37, 785-789. 